\title{
Neural Substrates of Alcohol-Induced Smoking Urge in Heavy Drinking Nondaily Smokers
}

\author{
Andrea King*,', Patrick McNamara', Michael Angstadt ${ }^{2}$ and K Luan Phan ${ }^{2,3}$ \\ 'Department of Psychiatry and Behavioral Neuroscience, The University of Chicago, Chicago, IL, USA; '2Department of Psychiatry, \\ University of Michigan, Ann Arbor, MI, USA; ${ }^{3}$ Mental Health Service, VA Ann Arbor Healthcare System, Ann Arbor, MI, USA
}

\begin{abstract}
A strong link exists between cigarette smoking and alcohol use, which may be explained by the experimental observation that alcohol ingestion promotes cigarette craving and precipitates smoking. At the neuroanatomic level, it is unclear where and how alcohol exerts these effects, although the process likely involves the ventral striatum given its function in motivational salience and appetitive reinforcement. In a double-blinded, placebo-controlled, crossover study, heavy drinking nondaily social smokers (ie, light smokers or 'chippers') were examined using functional magnetic resonance imaging after they ingested an acute dose of alcohol or placebo. We probed reactivity in the ventral striatum and other brain regions during exposure to visual smoking vs nonsmoking control cues. We found that alcohol enhanced self-reported ratings of desire to smoke, and in this context, significantly increased ventral striatum responses to smoking compared with control cues. In exploratory analyses, we observed that alcohol dampened orbitofrontal activity across both cue types, whereas dorsolateral prefrontal and anterior cingulate cortex activation to smoking cues was not affected by alcohol. This study bridges a pharmacological challenge approach to the study of brain reactivity to smoking cues, extends prior cigarette cue imaging studies to nondependent smokers, and elucidates a potential neurobiological mechanism to explain the co-consumption of alcohol and cigarettes in nondependent users.

Neuropsychopharmacology (2010) 35, 692-70I; doi:10.1038/npp.2009.177; published online II November 2009
\end{abstract}

Keywords: alcohol; $\mathrm{MMRl}$; ventral striatum; cues; smoking urge; nondaily smoker

\section{INTRODUCTION}

Tobacco and alcohol use are leading causes of preventable disease, and contribute to nearly seven million deaths each year worldwide (World Health Organization, 2007). A strong link exists between cigarette smoking and alcohol use (Dani and Harris, 2005; Harrison et al, 2008). Smokers are 10 times more likely to develop alcoholism than their nonsmoking counterparts (DiFranza and Guerrera, 1990). Relative to placebo, alcohol precipitates cigarette craving (Epstein et al, 2007) and increases cigarette consumption (Mitchell et al, 1995). Chronic alcohol use renders nicotine more reinforcing (Dani and Harris, 2005), which in turn may promote smoking behaviors and undermine attempts to quit. Despite such compelling epidemiological and experimental evidence, little is known about the neural mechanisms underlying concurrent and interactive use of alcohol and nicotine in humans (Sobell et al, 1990).

*Correspondence: Dr A King, Department of Psychiatry and Behavioral Neuroscience (MC-3077), University of Chicago, 584I S. Maryland Avenue, Chicago, IL 60637, USA, Tel: + 7737026181 , Fax: +773702 6454, E-mail: aking@bsdad.uchicago.edu

Received 25 June 2009; revised 18 September 2009; accepted 19 September 2009
Several recent functional neuroimaging studies have provided some initial clues on mechanisms underlying smoking or alcohol cue-induced brain reactivity. Smokers and drinkers are vulnerable to cravings when exposed to cues associated with the pleasures of smoking and drinking (eg, cigarette-related images, olfactory cues) (Niaura et al, 1988; Wrase et al, 2002; Brody, 2006; Bragulat et al, 2008). When exposed to smoking-related cues, smokers have been shown to exhibit robust responses in mesolimbic brain regions (nucleus accumbens (NAcc) and broader ventral striatum (vSTR)) (David et al, 2005; Franklin et al, 2007). The vSTR is centrally implicated in brain-based models as the common pathway of addiction (Robinson and Berridge, 1993; Koob and Le Moal, 2001; Everitt and Robbins, 2005; Kalivas and Volkow, 2005). Thus, there is reason to predict that modulating vSTR function would impact the desire and motivation to use nicotine and other addictive substances (Kalivas and Volkow, 2005) given that vSTR is thought to signal the presence of nicotine in the environment. In humans, the vSTR is activated by smoking-related cues (David et al, 2005; Brody, 2006) and associated with abstinence-induced craving (Wang et al, 2007). Further, acute alcohol administration enhances vSTR activity (Gilman et al, 2008) and its reactivity to alcoholic drink odors (Bragulat et al, 2008). Given that alcohol is often consumed along with cigarette smoking, alcohol may serve 
as a potent amplifier of VSTR reactivity to salient smokingrelated cues.

We have previously shown that alcohol intensifies smoking urges in nondependent, heavy drinking social smokers (King and Epstein, 2005; Epstein et al, 2007). Social smokers, also known as light smokers, nondaily smokers, or tobacco chippers, appear to be increasing in prevalence (for review, see Shiffman, 2009), and are able to smoke without experiencing significant withdrawal symptoms or associated consequences (Shiffman, 1989; Shiffman et al, 1990, 1995). Nondaily social smokers also tend to exhibit frequent pairings and exacerbations of cigarette smoking in the context of alcohol drinking (King et al, 2008). In terms of neuroimaging research, this subgroup may be of interest due to lack of the potential confound of withdrawal and abstinence states that may increase amplitude in frontal brain regions (McClernon et al, 2005; Wang et al, 2007) and the reduced potential for 'ceiling effects' in smoking urge ratings (Sayette et al, 2000, 2005; Epstein et al, 2007). However, to our knowledge, there have been no neuroimaging studies to date assessing non-nicotine dependent smokers' response to cigarette cues and the potential enhancing effects of alcohol on such responses. Therefore, this study was a preliminary investigation designed to characterize the brain's, particularly the vSTR, response to smoking cues in the context of consuming an intoxicating dose of alcohol or placebo in heavy drinking nondaily smokers. We hypothesized that alcohol would amplify reactivity of vSTR to smoking-related cues during this vulnerable period of heightened craving. We also explored alcohol's effects on other brain regions that have been shown to be activated by smoking cues, including anterior and posterior cingulate cortex (ACC, PCC), orbitofrontal cortex (OFC), and dorsolateral prefrontal cortex (DLPFC) (Brody et al, 2002; Due et al, 2002; David et al, 2005; McClernon et al, 2005, 2008a, b; Wilson et al, 2005; McBride et al, 2006; Franklin et al, 2007).

\section{MATERIALS AND METHODS}

\section{Subjects}

Twelve healthy, right-handed young adults (age 21-26 years) participated after giving written informed consent as approved by the University of Chicago Institutional Review Board. To be included, subjects had to be nondependent, light social smokers (smoke on a nondaily basis, between 1 and 50 cigarettes per week) with nondependent heavy social drinking patterns (consuming $\geqslant 10$ alcoholic drinks per week while engaging in 1-5 weekly heavy or 'binge' drinking (5+ drinks per occasion for men; $4+$ drinks for women; SAMHSA, 2005) episodes). These specific criteria were chosen to be consistent with prior studies, indicating these social drinkers-smokers exhibit sensitivity to alcoholinduced smoking urges (King and Epstein, 2005; Epstein et al, 2007; King et al, 2008) and to be consistent with epidemiological studies examining smoking patterns in at-risk alcohol drinkers (Dawson, 2000; SAMHSA, 2005). All smoking and drinking criteria were determined by TimeLine Follow-Back (Sobell et al, 1979; Sobell and Sobell, 1995) and Quantity-Frequency Interview indices (Cahalan and Cisin, 1968). Exclusion criteria included past or current substance, alcohol, or nicotine dependence, as well as other major psychiatric, neurological, or medical illness as confirmed by medical examination, the Fagerström Test of Nicotine Dependence (FTND; Heatherton et al, 1991), and a Structured Clinical Interview for DSM-IV (First et al, 1995) conducted by a trained diagnostic interviewer. Excluding candidates with alcohol or nicotine dependence avoided potential abstinence and withdrawal complications or confounding effects on functional magnetic resonance imaging (fMRI) signal.

\section{Experimental Protocol}

The study used a randomized, blinded, two-session, withinsubjects, double-dummy design. The two sessions were separated by at least $48 \mathrm{~h}$ and involved an adapted version of a smoking-cue fMRI task previously used (Brody, 2006). Before each session, participants were instructed to abstain from alcohol, recreational drugs, and any psychoactive medications for at least $48 \mathrm{~h}$, as well as caffeine, food, and cigarette smoking for $3 \mathrm{~h}$. On arrival, between 11:00 a.m - 3:00 p.m, the subject underwent abstinence verification, consumed a low-fat snack (20\% daily calories), and acclimated to the laboratory. During this period, to minimize potential expectancy effects that might dampen urges and cue-related activation (Wilson et al, 2005; McBride et al, 2006), the subjects were informed that they may or may not be allowed to smoke later in the session; however, in all cases, no smoking occurred during the session.

Approximately $45 \mathrm{~min}$ after arrival, the participant was served a beverage and a small, placebo gel capsule (containing dextrose). To reduce alcohol expectancies, the subject was told that both the beverage and gel-capsule might contain alcohol, a stimulant, a sedative, a placebo, or a combination of these substances. The beverage only actually contained either alcohol $(0.8 \mathrm{~g} / \mathrm{kg} ; 16 \%$ volume alcohol) or placebo $(0.0 \mathrm{~g} / \mathrm{kg} ; 1 \%$ volume ethanol as a taste mask). The beverages were prepared with Kool-Aid, water, Splenda, and the appropriate dose of 190-proof ethanol based on body weight and were consumed through a straw in a lidded, opaque cup to conceal potential scent cues. Women received an $85 \%$ dose to adjust for total body water differences (Sutker et al, 1983; Frezza et al, 1990). The subject consumed the beverage over a 13 min interval, timed such that the upcoming fMRI task would concur with expected peak blood alcohol levels and subjective effects such as smoking urge (Epstein et al, 2007). Immediately after beverage consumption, the subject was escorted into the scanning room and underwent approximately $20 \mathrm{~min}$ of fMRI preparation and structural scanning before beginning the fMRI task.

The fMRI task involved viewing pictorial stimuli of smoking and control content as performed previously (Due et al, 2002; McClernon and Gilbert, 2004; David et al, 2005; McClernon et al, 2005, 2008b; Brody, 2006). In brief, stimuli consisted of color photographs of smoking content $(n=64$; images of a person, holding a cigarette, lighting a cigarette, etc) or control content ( $n=64$; similar to the smoking-related images, but with a person handling neutral, nonsmoking cue items (ie, pens, markers, glasses)) (see Figure 1). The stimulus set was 


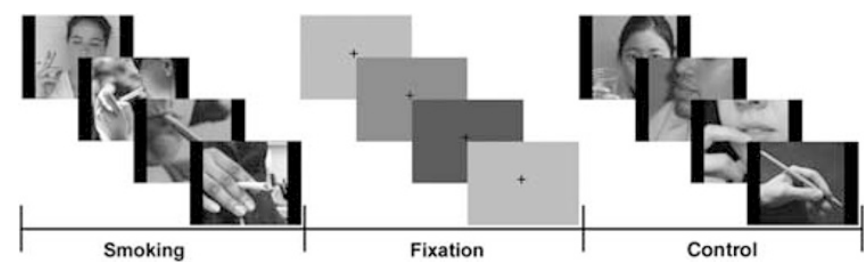

Figure I Schematic illustration of $\mathrm{fMRI}$ task and stimuli. Participants were presented to visual cues (smoking and control) in $20 \mathrm{~s}$ blocks that were interspersed with blank/fixation blocks.

composed of images obtained from the International Smoking Image Series (Gilbert and Rabinovich, 2003) $(n=51)$, a proprietary set $(n=77)$, and four blank grayscale images with a centered fixation cross. The image types were matched for gender, race, environment, general complexity, and RGB color value. The images were presented in $20 \mathrm{~s}$ blocks (four per block) without repetition. Picture blocks were interspersed with $20 \mathrm{~s}$ blocks of four blank, gray-scale screens with a central fixation crosshair. To orient subjects and maintain attention to task, we instructed subjects to 'imagine yourself in each of the following images' and press a button for each new image; participants' reaction time to each image was collected. The entire paradigm consisted of four fMRI 'runs' for a total task time of $22 \mathrm{~min}$.

\section{Functional Imaging: Acquisition and Analysis}

Blood oxygenated level-dependent (BOLD) signals were collected using a $3 \mathrm{~T}$ GE MRI scanner from 30 axial, 5 -mm-thick slices using a $\mathrm{T} 2{ }^{\star}$-sensitive gradient echo reverse spiral acquisition sequences (repetition time, $2000 \mathrm{~ms}$; echo time, $25 \mathrm{~ms} ; 64 \times 64$ matrix; $24 \mathrm{~cm}$ field of view; flip angle, $77^{\circ}$ ). This was followed by a high-resolution, T1-weighted volumetric anatomical scan for anatomical localization.

Data from all 24 sessions met criteria for high quality with minimum head motion $(<1 \mathrm{~mm}$ displacement in any one direction). Functional data were analyzed using SPM2 (Welcome Department of Cognitive Neurology, London, UK; http://www.fil.ion.ucl.ac.uk/spm) using a pharmacological fMRI and region of interest (ROI)-based analytic approach described previously (Phan et al, 2008). In brief, images were spatially realigned to correct for head motion, warped to a canonical template in Montreal Neurologic Institute (MNI) space, resampled to $2 \mathrm{~mm}^{3}$ voxels, and smoothed with an $8 \mathrm{~mm}^{3}$ kernel to minimize noise and residual differences in gyral anatomy. The general linear model was applied to the time series, convolved with the canonical hemodynamic response function and with a $128 \mathrm{~s}$ high-pass filter. Condition effects were modeled with box-car regressors (smoking, control, fixation) representing the occurrence of each block type, and effects were estimated at each voxel and for each subject. A statistical image for the contrast of smoking $>$ fixation and control $>$ fixation was obtained for each cue type and analyzed in a second-level random-effects model for significant differences in brain activation between the alcohol (ALC) and placebo (PBO) sessions.

In this multifactorial mixed-model, repeated-measures ANOVA, beverage (ALC or PBO) and cue (smoking or control) were fixed factors, and subject was a random factor. We tested for significant main effect of beverage, main effect of cue, and interaction of beverage $\times$ cue across the entire brain. The threshold for significance for activations observed in the VSTR was set to stringent level of $p<0.05$, false discovery rate, which effectively corrects for multiple voxel-wise comparisons across the entire brain (Genovese et al, 2002). In our exploratory analyses, to compare results with prior smoking cue-induced studies (Brody et al, 2002; Due et al, 2002; David et al, 2005; McClernon et al, 2005, 2008a, b; Wilson et al, 2005; McBride et al, 2006; Franklin et al, 2007) and alcohol challenge fMRI studies (Bragulat et al, 2008; Gilman et al, 2008) and for subsequent generation of new hypotheses, we report activation results in other brain regions other than the vSTR that survive the more lenient threshold of $p<0.001$ (uncorrected) with an extent threshold of clusters with a minimum of 10 contiguous voxels. All activation loci were determined by anatomically based labels (Walter et al, 2003) based on the atlas of Tzourio-Mazoyer et al (2002). As there is no consensus on an atlas-based anatomical ROI or automated anatomical labeling method for clearly defining the vSTR, we used published studies to locate if observed activation would fall within the 'vSTR' as previously described (David et al, 2005; Martinez et al, 2003). David et al (2005) showed that greater ventral striatal activation in addicted smokers than nonsmokers presented with smoking-related cues using fMRI, and defined the vSTR by defining anatomical landmarks that are consistent with the activation foci observed in our study.

To clarify main effects of beverage, cue, or their interactions observed from the whole-brain voxel-wise ANOVA, we conducted post hoc $t$-tests to determine the source of significance. Within our a priori ROI (vSTR), we extracted BOLD signal responses (parameter estimates, $\beta$ weights) to smoking and control cues from a functional ROI defined as $10 \mathrm{~mm}$ spheres (left, right) surrounding the peak activation, and entered these values into a repeatedmeasures ANOVA where beverage (ALC or PBO) and cue (smoking or control) were fixed factors, and subject was a random factor. Significant main effect of beverage and of cue and beverage $\times$ cue interactions were followed with post hoc paired $t$-tests on ROI-derived $\beta$ weights to determine the source of significance; significance was set at $p<0.05$, two-tailed.

\section{Behavioral Data Analysis}

Self-reported changes in smoking urge were assessed over the course of the protocol (immediately before consumption, and then repeated at $30,45,60$, and $75 \mathrm{~min}$ after initiation of alcohol/placebo ingestion) using scores from the first item of the Brief Questionnaire on Smoking Urges (BQSU; Cox et al, 2001). This item ('I have a desire for a cigarette right now') was rated from 1 to 7 (increasing intensity), has been shown to be influenced by alcohol (King and Epstein, 2005; Epstein et al, 2007), and is significantly correlated with the total BQSU score $(r=+0.91)$ (Epstein et al, 2007). These were analyzed using two-factor ANOVA with factors beverage (ALC, PBO) and time. Significant main effect of beverage and beverage by time interactions were followed with paired $t$-tests (ALC $v s \mathrm{PBO}$ ) to examine 
the source of significance at each time point. We determined the effects of ALC on fMRI task performance in terms of response times (in milliseconds) using a beverage (ALC, PBO) and cue (smoking, control) ANOVA; any significant main effects or beverage $\times$ cue interactions were followed up with paired $t$-tests (ALC vs PBO). Significance for all tests was set at $p<0.05$, two-tailed.

\section{RESULTS}

\section{Description of Study Population}

The sample was composed of 10 men and 2 women, with an average age of 23.2 years $( \pm 1.8 \mathrm{SD}$ ) and a racial breakdown of 8 Caucasians, 3 Asians, and 1 African-American subject. Of the 12 subjects, 11 (92\%) met lifetime criteria for DSMIV alcohol abuse, but none met lifetime criteria for either alcohol or nicotine dependence. As expected, FTND scores were fairly negligible $(M=0.08 \pm 0.08$; one subject scored a 1 on this scale, all others 0 ), confirming lack of physical nicotine dependence in the sample. The Time-Line FollowBack interview of participants' past month alcohol drinking and cigarette smoking behaviors revealed that all subjects had nondaily patterns for both alcohol drinking and cigarette smoking. On average, alcohol drinking occurred on 13.2 $( \pm 7.1)$ days in the past month, with $7.8( \pm 3.4)$ heavy drinking days. Cigarette smoking occurred on $14.7( \pm 7.1)$ of days in the past month. Smoking co-occurred on $71 \%$ (9.4/13.2) of the days in which any alcohol was consumed, and $83 \%(6.5 / 7.8)$ of heavy drinking days. The average number of drinks consumed per drinking occasion was $6.7( \pm 3.5)$ and the average number of cigarettes smoked per smoking day was 4.3 ( \pm 2.8$)$. The average last self-reported last alcohol use before the study sessions was $3.8( \pm 1.7)$ days and the average last cigarette use was 2.7 ( \pm 2.3$)$ days. All baseline breathalyzer tests were negative for presence of alcohol, and baseline exhaled carbon monoxide levels were negligible ( $\leqslant 2.4 \pm 1.8$ p.p.m.), confirming compliance with study abstinence instructions on the testing days.

\section{Alcohol Effects on BAC, Smoking Urge, and Task Performance}

BAC levels for the session are depicted in Figure 2a. Alcohol ingestion increased $\mathrm{BAC}$ to a peak of $0.90 \mathrm{mg} \%$ at $75 \mathrm{~min}$ after initiation of the beverage (ie, $60 \mathrm{~min}$ after drinking completion), with a slow elimination phase over the next few hours. For intensity of desire to smoke ratings, a main effect of beverage $\left(\mathrm{F}_{(1,11)}=7.47, p<0.019\right)$ and time $\left(\mathrm{F}_{(4,44)}=15.89, p<0.000\right)$ was observed (Figure $\left.2 \mathrm{~b}\right)$. Specifically, predrink baseline scores did not differ between the sessions ( $\left(\right.$ ALC $2.9( \pm 1.7)$ vs PBO $2.8( \pm 1.4) ; t_{(11)}=0.13$, $p=$ not significant), or at $30 \mathrm{~min}$ after the initiation of the beverage ingestion $(3.3( \pm 2.1)$ vs 2.9 ( \pm 1.7$)$, respectively; $t_{(11)}=0.63, p=$ not significant). However, alcohol, compared with placebo, did increase ratings of desire to smoke at both $45 \mathrm{~min}$ (ALC $4.8( \pm 1.7)$ vs $\mathrm{PBO} \quad 3.7 \quad( \pm 1.8)$; $\left.t_{(11)}=2.24, p<0.05\right)$ and $60 \mathrm{~min}$ after drinking initiation $\left(5.2( \pm 1.2)\right.$ vs $4.0( \pm 1.7)$, respectively; $\left.t_{(11)}=2.45, p<0.05\right)$, and persisted at 75 and $90 \mathrm{~min}$. Of note, fMRI scanning occurred between 35 and $55 \mathrm{~min}$ after drinking initiation, corresponding to the ascending limb of the BAC and
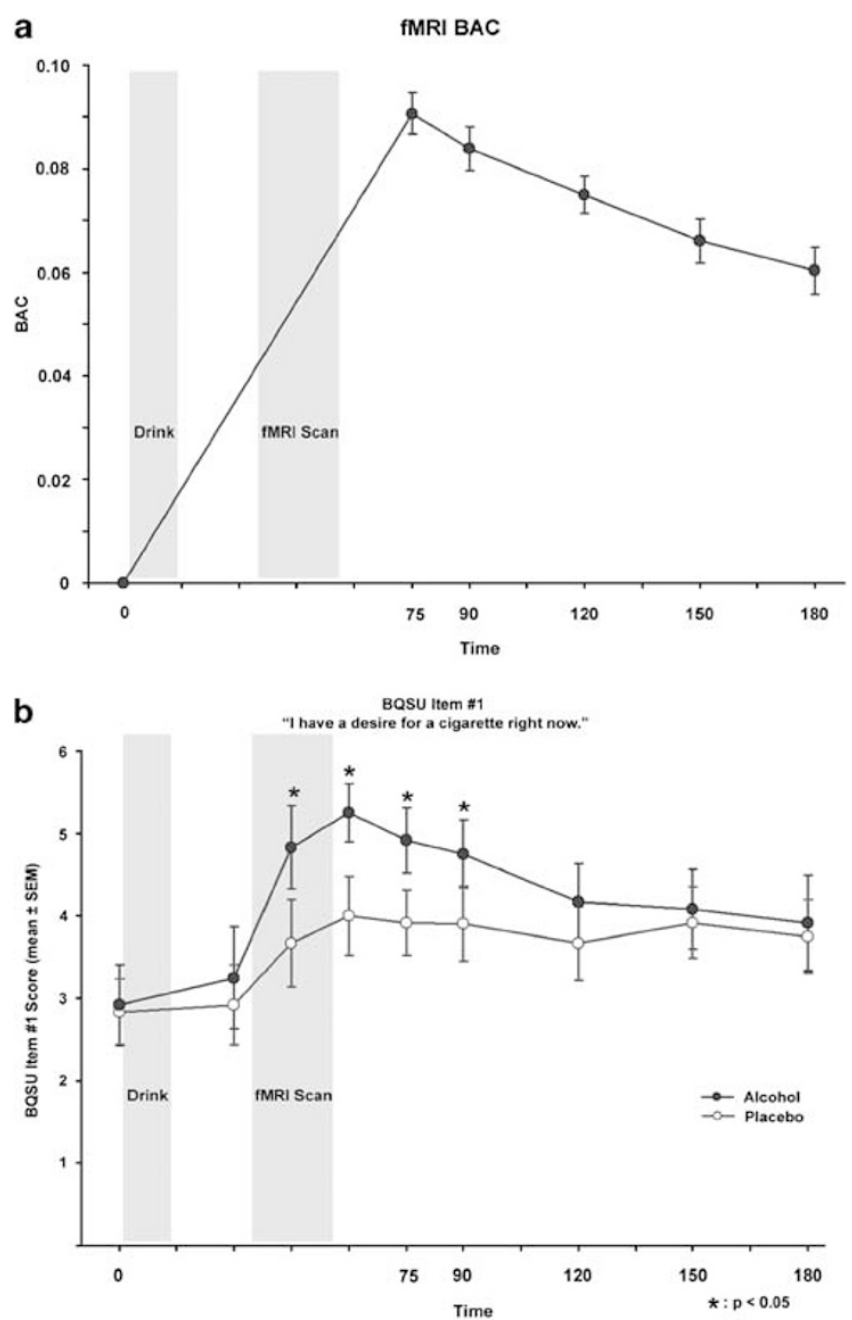

Figure 2 (a) Alcohol effects on breath alcohol levels throughout the session. 'Drink' shade corresponds to the timing and duration of alcohol/ placebo ingestion, and ' $\mathrm{MMRI}$ scan' corresponds to the timing and duration when brain activation was measured. (b) Alcohol effects on self-reported desire to smoke ratings. Alcohol elicited greater smoking urge (> placebo) at 45,60 , and 75 min after initiation of beverage consumption. 'Drink' shade corresponds to the timing and duration of alcohol/placebo ingestion, and ' $\mathrm{M} M \mathrm{RI}$ scan' corresponds to the timing and duration when brain activation was measured. $* p<0.05$, two-tailed.

smoking urge intensity. Finally, in terms of response times for button pressing on onset of new stimuli during the fMRI tasks, there were no significant effects of beverage, cue type, or interactions of these variables (all $p_{\mathrm{s}}>0.30$ ).

\section{Alcohol Effects on Ventral Striatum Activity}

As expected, we observed that alcohol increased activity in both left and right vSTR as shown by a significant main effect of beverage (ALC $>$ PBO) (see Table 1 and Figure 3 ). It is important to note that these activations produced by alcohol survived correction for multiple comparisons across the entire brain. ROI-based analyses of vSTR activity showed that alcohol, compared with placebo, enhanced activity in the right vSTR in the presence of the smoking cues vs control cues (beverage $\times$ cue, $p=0.01$; post hoc $t$-tests: smoking cue, alcohol $>$ placebo, $p=0.003$; alcohol, 
Table I Main Effects of Session, Cue, and Their Interactions, on Brain Response

\begin{tabular}{|c|c|c|c|c|c|c|c|c|}
\hline \multirow{2}{*}{$\begin{array}{l}\text { ANOVA } \\
\text { Main effect }\end{array}$} & \multirow{2}{*}{$\begin{array}{l}\text { Post hoc t-test } \\
\text { Direction }\end{array}$} & \multirow[b]{2}{*}{ Region } & \multirow[b]{2}{*}{ Laterality } & \multirow{2}{*}{$\begin{array}{c}\text { Cluster } \\
\text { size }\left(\mathrm{mm}^{3}\right)\end{array}$} & \multirow[b]{2}{*}{$Z$ score } & \multicolumn{3}{|c|}{ MNI Coordinates } \\
\hline & & & & & & $(x$, & $y$, & z) \\
\hline \multirow[t]{17}{*}{ Session } & $\mathrm{PBO}>\mathrm{ALC}$ & Middle occipital gyrus & $\mathrm{R}$ & 10040 & 5.40 & 46 & -82 & 0 \\
\hline & & & $\mathrm{L}$ & 504 & 3.60 & -28 & -92 & 6 \\
\hline & & & $\mathrm{R}$ & 720 & 3.87 & 20 & -94 & 32 \\
\hline & & & $\mathrm{R}$ & 408 & 3.85 & 14 & -104 & 18 \\
\hline & & & $\mathrm{R}$ & 1232 & 3.68 & 2 & -92 & 6 \\
\hline & & & $\mathrm{R}$ & 392 & 3.61 & 4 & -72 & 12 \\
\hline & & Precuneus & $L$ & 2392 & 4.26 & -14 & -88 & 48 \\
\hline & & Postcentral gyrus & $\mathrm{L}$ & 200 & 3.52 & -30 & -24 & 44 \\
\hline & & Parahippocampal gyrus & $\mathrm{R}$ & 112 & 3.77 & 22 & -44 & 4 \\
\hline & & & $L$ & 104 & 3.44 & -16 & -16 & -10 \\
\hline & & Gyrus rectus/OFC & $\mathrm{R}$ & 376 & 3.74 & 6 & 58 & -16 \\
\hline & & Superior frontal gyrus/OFC & $\mathrm{R}$ & 544 & 3.50 & 2 & 36 & -10 \\
\hline & & Cerebellum & $\mathrm{R}$ & 208 & 3.63 & 30 & -32 & -32 \\
\hline & & Posterior cingulate gyrus & $L$ & 184 & 3.49 & -14 & -36 & 26 \\
\hline & & Inferior frontal gyrus & $L$ & 184 & 3.39 & -44 & 28 & 4 \\
\hline & & Superior occipital gyrus & $\mathrm{L}$ & 160 & 3.30 & -10 & -104 & 12 \\
\hline & $\mathrm{ALC}>\mathrm{PBO}$ & Middle frontal gyrus & $\mathrm{R}$ & 2304 & 4.65 & 38 & 16 & 50 \\
\hline \multirow[t]{7}{*}{ Cue } & $S>C$ & Middle frontal gyrus/DLPFC & $\mathrm{R}$ & 680 & 3.86 & 36 & 38 & 38 \\
\hline & & & $\mathrm{L}$ & 616 & 3.84 & -32 & 38 & 34 \\
\hline & & Anterior cingulate gyrus & $\mathrm{R}$ & 184 & 3.59 & 2 & 32 & 6 \\
\hline & & Posterior cingulate gyrus & $\mathrm{R}$ & 152 & 3.43 & 8 & -30 & 50 \\
\hline & $N>C$ & Inferior occipital gyrus & $\mathrm{R}$ & 1336 & 3.74 & 26 & -96 & -6 \\
\hline & & & $L$ & 248 & 3.26 & -26 & -98 & -16 \\
\hline & & Fusiform gyrus & $\mathrm{R}$ & 344 & 3.60 & 28 & -36 & -22 \\
\hline \multirow[t]{7}{*}{ Interaction } & $\begin{array}{l}(A L C C \& P B O S)> \\
(A L C S \& P B O C)\end{array}$ & Middle frontal gyrus & $L$ & 640 & 3.99 & -20 & 18 & 44 \\
\hline & & Mid cingulate gyrus & $\mathrm{R}$ & 1256 & 3.94 & 12 & -10 & 42 \\
\hline & & & $\mathrm{R}$ & 224 & 3.55 & 12 & 8 & 36 \\
\hline & & Precentral gyrus & $\mathrm{R}$ & 2968 & 3.90 & 26 & -28 & 58 \\
\hline & & & $\mathrm{L}$ & 232 & 3.33 & -22 & -26 & 58 \\
\hline & & Cerebellum & L & 184 & 3.69 & -28 & -70 & -40 \\
\hline & & Putamen & $L$ & 104 & 3.28 & -24 & 0 & 0 \\
\hline
\end{tabular}

ALC, alcohol; PBO, placebo; S, smoking; C, control; DLPFC, dorsolateral prefrontal cortex; mPFC, medial prefronal cortex; OFC, orbitofrontal cortex; MNI, Montreal Neurologic Institute.

${ }^{a} \mathrm{~A}$ priori region of interest in bold; Significant at $p<0.05$, false discovery rate (FDR)-corrected for multiple voxel-wise comparisons across the entire brain. All other activations shown at $p<0.00$ I (uncorrected) with a cluster extent threshold of at least 10 contiguous voxels. 
smoking cue $>$ control cue, $(p=0.04)$ ) (Figure 4). Alcohol ( $>$ placebo) had a similar effect on smoking cues in the left vSTR; although the beverage $\times$ cue interaction was not significant, we did observe increased activation in left vSTR only during smoking cues $\left(t_{(11)}=2.37 p=0.04\right)$ and not during control cues.

\section{Relationship Between Striatal Activation and Smoking Urge}

We conducted an exploratory correlation analysis to examine the relationship between alcohol-induced change

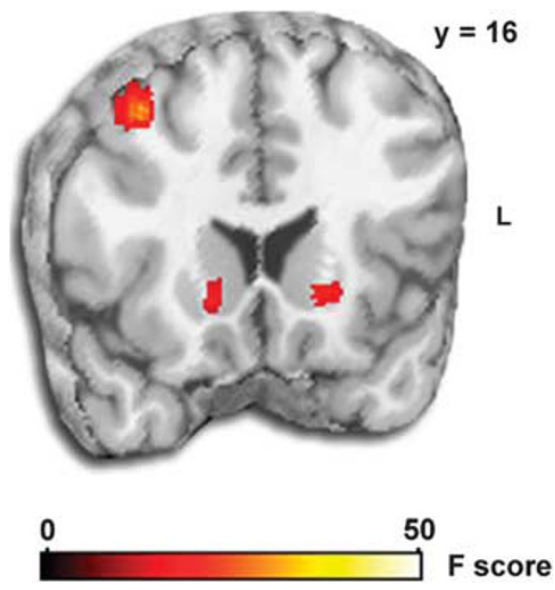

Figure 3 Alcohol effects on ventral striatum. Statistical F map (wholebrain voxel-wise repeated-measures ANOVA) overlaid on a canonical brain rendering (Montreal Neurologic Institute $(\mathrm{MNI})$ coronal y plane $=16$ ) showing a main effect of session (alcohol, placebo) on bilateral ventral striatum activation. in craving ( $\mathrm{ALC}>\mathrm{PBO}$ change in extent of subjective smoking urge during the 30 and $45 \mathrm{~min}$ post-beverage ingestion time points) and change activation (ALC $>$ PBO) in left and right vSTR. As would be expected, we observed a significant positive correlation between change in right vSTR and change in urge at the $30 \mathrm{~min}$ time point $(r=+0.61$, $p=0.03$ ); however, correlations at the $45 \mathrm{~min}$ time point $(r=+0.30, p=$ not significant) and in the left vSTR at either time point were not significant $(r=+0.41, r=+0.11$, $p=$ not significant, respectively).

\section{Alcohol Effects on Activity in Other Brain Regions}

Our exploratory whole-brain voxel-wise analyses revealed a main effect of beverage (across both cue types) on brain activation in medial OFC, cuneus, precuneus, fusiform gyrus, middle and inferior frontal gyrus, mid cingulate cortex, middle and superior occipital gyrus, middle temporal gyrus, parahippocampal gyrus, cerebellum and postcentral gyrus (see Table 1). Post hoc t-tests showed that alcohol attenuated (PBO $>$ ALC) activity in OFC, middle and superior occipital gyrus, pre/cuneus, fusiform gyrus, parahippocampal gyrus, posterior cingulate gyrus, and inferior frontal gyrus, whereas it enhanced (ALC $>\mathrm{PBO}$ ) activity in middle frontal gyrus and middle temporal gyrus. A main effect of cue type across beverage was observed in perigenual ACC, PCC, DLPFC, fusiform gyrus, and inferior occipital gyrus. Post hoc t-tests showed that smoking ( $>$ control) cues elicited greater activity in DLPFC, ACC, and PCC, whereas control ( $>$ smoking) cues increased activity in fusiform gyrus and inferior occipital gyrus. Significant beverage $\times$ cue interactions were observed in middle cingulate cortex, middle frontal gyrus, precentral gyrus, cerebellum and putamen (see Table 1).

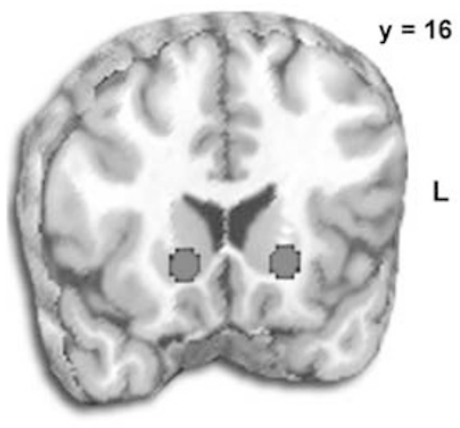

b

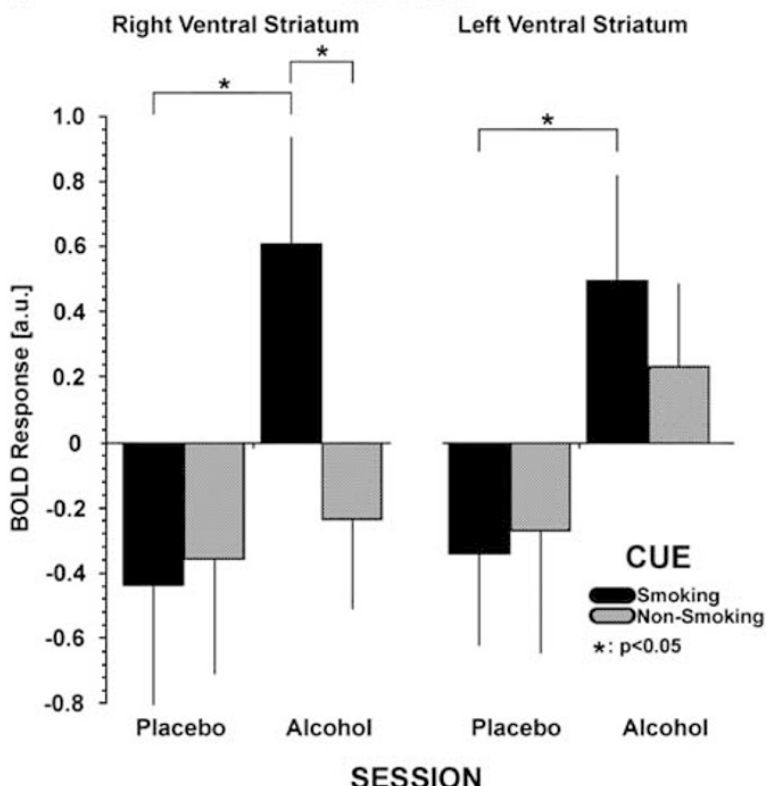

Figure 4 (a) Brain image illustrating the functional ROI from which data were extracted. (b) Mean $\beta$ weights ( \pm SEM) extracted from right and left ventral striatum, with a significant beverage $\times$ cue interaction in the right ventral striatum to smoking cues during the alcohol session, $* p<0.05$, and post hoc exploratory t-test showing greater left vSTR activity to smoking cues on the alcohol vs placebo condition, ${ }^{*} p<0.05$. 


\section{DISCUSSION}

We found that consumption of a moderately intoxicating oral dose of alcohol increased ratings of desire to smoke, even within the constraints of the fMRI scanning environment. Moreover, we observed that alcohol amplified vSTR reactivity to appetitive cues associated with smoking in young individuals who tend to use cigarettes in the context of alcohol intoxication. Taken together, these findings may represent a critical initial step to understanding why alcohol and cigarettes are often consumed together (Dani and Harris, 2005).

In our prior studies, we have shown that alcohol dosedependently increases urge to smoke even in the absence of in vivo smoking cues in heavy social drinking, nonnicotine-dependent smokers (King and Epstein, 2005; Epstein et al, 2007). In those laboratory studies examining two independent samples, heavy drinking social smokers were found to crave cigarettes more after consuming an intoxicating alcohol dose (4-5 drink equivalent) compared with a lighter alcohol dose (2-3 drink equivalent) or a placebo beverage, and this effect was driven by heightened alcohol-related stimulation rather than as a means to counteract alcohol's sedative effects (Epstein et al, 2007). These data suggest that nondependent smokers are likely to have smoking urges when drinking, and we may speculate that such urges would lead to escalation of their smoking behaviors in the context of alcohol intoxication. However, until recently, these studies were confined to self-report or behavioral studies, with little neurobiological evidence to explain the mechanism by which alcohol stimulates smoking urges and/or behaviors within intoxicated smokers.

Emerging evidence from functional neuroimaging has consistently shown that visual and olfactory cues associated with drug use (nicotine, alcohol, etc) evoke robust activations in mesolimbic NAcc and vSTR, even in nondependent users (Kareken et al, 2004; David et al, 2005; Franklin et al, 2007). Moreover, vSTR metabolism and reactivity to appetitive, smoking cues is exaggerated in smokers and correlated with cigarette craving (David et al, 2005; McClernon et al, 2005; Wang et al, 2007). Interestingly, alcohol enhances vSTR activity to emotional stimuli and self-ratings of intoxication predict striatal activation (Gilman et al, 2008). Convergent with these findings, our data show that alcohol intoxication amplifies vSTR reactivity to smoking-related cues in the context of cigarette craving. Together, these data fit well with current neurobiological models that implicate vSTR as a central and common node of dysfunction in drug addiction (Koob and Le Moal, 1997; Everitt and Robbins, 2005; Kalivas and Volkow, 2005). Future studies are needed to delineate the neurochemical underpinnings of alcohol's effects on reward/cue-related vSTR function, though some evidence suggests that enhanced release of dopamine is involved (Knutson and Gibbs, 2007). It is plausible that alcohol promotes smoking behaviors (drug seeking) by enhancing the motivational salience of drug cues (smoking-related images) by reward-related neural substrates (ie, dopamine release, vSTR activation). Although the neurochemical mechanism by which alcohol might enhance vSTR activation is unclear, we would speculate that alcohol may promote dopamine release preferentially in the vSTR/NAcc as shown by a recent $\left[{ }^{11} \mathrm{C}\right]$ raclopride positron emission tomography study in humans (Boileau et al, 2003). Interestingly, alcohol drinking in alcohol-preferring rats enhances dopamine release in mesolimbic brain areas including the vSTR (Honkanen et al, 1997; Bustamante et al, 2008). This increase in dopamine may be reflected by the increase in the BOLD fMRI signal in vSTR (Knutson and Gibbs, 2007). In addition, it is possible that alcoholmediated opioid release in vSTR may relate to this pathway (Barson et al, 2009), and/or to increased motivation to continued drinking or smoking behavior.

In this study, we also observed that alcohol attenuated activity in OFC regardless of cue type, whereas alcohol did not affect the smoking cue-related activation in the DLPFC, ACC, and PCC. The OFC has been shown to mediate adaptive behavior in the context of changing rewardpunishment contingencies (Rogers et al, 1999; O'Doherty et al, 2001; Ernst et al, 2002). Moreover, OFC has been hypothesized to mediate drug craving and motivational value of a stimulus in the context of impulse control (London et al, 2000). Nicotine-dependent individuals show increased OFC activity in response to smoking cues (Brody et al, 2002; David et al, 2005). Further, recently, Brody et al (2007) showed that the orbital medial frontal gyrus, among other prefrontal areas, is engaged when smokers attempt to resist craving when processing cigarette cues. Among heavy, nicotine-dependent smokers, DLPFC, ACC, and PCC are consistently activated by smoking cues and the extent of activation these regions has been shown to be positively correlated with smoking craving/urge (David et al, 2005; McClernon et al, 2005; McBride et al, 2006; Franklin et al, 2007; Wang et al, 2007). This study represents the first extension to alcohol preadministration of such effects, and the data suggest that smoking cue-related reactivity in these prefrontal regions is not altered by alcohol, at least within heavy drinking nondaily social smokers.

The advantages of including participants with nondependent smoking and drinking patterns include avoiding potential confounds of withdrawal and abstinence states that may increase amplitude in frontal brain regions (McClernon et al, 2005; Wang et al, 2007) and potential 'ceiling effects' in smoking desire ratings (Sayette et al, 2000, 2005; Epstein et al, 2007). These persons are also of interest because they exhibit frequent pairings of cigarette smoking in the context of alcohol drinking (King et al, 2008), and are able to smoke on a non daily basis without experiencing overt consequences, withdrawal symptoms, or complexities in reward or punishment-based decisionmaking (Shiffman, 1989; Shiffman et al, 1990, 1995). In this study, we may speculate that neural activation in regions associated with motivational salience (striatum) underlie alcohol's stimulant-like and smoking-urge increasing effects that lead to chronic, (Epstein et al, 2007), reciprocal drinking-smoking co-misuse (McKee et al, 2004; Harrison et al, 2008). It is important to note that in this study, cigarette cues alone (ie, in the placebo session) did not activate the striatum, which may be consistent with the behavioral patterns in these subjects. As mentioned earlier, heavy drinking nondaily smokers desire cigarettes primarily in the presence of alcohol, especially during heavy or binge drinking episodes. Therefore, for persons with nondependent smoking and drinking patterns, we might 
predict that dopamine-mediated conditioned responses in regions relating to motivational salience would not be pervasive, but more selective for alcohol. Future studies examining neurobiological processes in addicted smokers at various developmental stages of initiation, exacerbation, and maintenance/recovery phases $v s$ those with sustained nondaily patterns may help elucidate the function of motivational processes and cue reactivity in these various stages.

There are several strengths to note in this study, including a within-subjects design for beverage (alcohol, placebo) and cue type (smoking, control), validation of paradigm features (salience of smoking cues and alcohol as a potent stimulus for cigarette urges), and minimization of expectancy effects of cigarette availability (Wilson et al, 2005). However, there are also several limitations worth mentioning, including (1) small sample size precluded ascertainment of the function of individual difference factors, such as effects related to sex (King et al, 2008, 2009), race/ethnicity, or differential alcohol and cigarette use patterns (Smolka et al, 2006; McClernon et al, 2008b); (2) reliance on self-report for smoking urge assessment, and not including other measures, such as peripheral skin conductance or heart rate; (3) inability to examine dose-response relationships or threshold effects of alcohol (King and Epstein, 2005; Epstein et al, 2007) due to inclusion of only one dose of alcohol. Finally, alcohol exerts its reinforcing effects on multiple neurotransmitter systems in the brain making it difficult to ascertain a specific neurochemical pathway for the effects in this fMRI study; however, recent data suggest that vSTR release of dopamine, the key reward signal, increases local BOLD signal by postsynaptic dopamine receptor activation (Knutson and Gibbs, 2007) and reward-related vSTR activation is predicted by extent of dopamine release (Schott et al, 2008). Future studies are needed to clarify this issue further and to extend these findings to populations both at heightened risk for and with alcohol and nicotine dependence.

In conclusion, this study bridged a pharmacological challenge approach to the rapidly emerging study of brain reactivity to drug cues and elucidates a potential neurobiological mechanism to explain the co-consumption of alcohol and cigarettes. Results showed that the vSTR reacts to drugrelated visual cues only under the influence of alcohol but not placebo in persons who engage in regular substance co-misuse. This observation was concurrent to the increase in subjective urge to smoke induced by alcohol, and the increase in right ventral striatal activation was associated with increased smoking urge induced by alcohol. Clinically, these results may help to understand mechanisms underlying alcohol-related increases in smoking relapse in those attempting to quit smoking but who still drink alcohol (Baer and Lichtenstein, 1988; Dawson, 2000; Harrison et al, 2008), and to target neurobiological strategies to avert perpetuation of concomitant heavy drinking and cigarette smoking behaviors in adolescents and emerging adults at risk for development of dependence on either or both substances.

\section{ACKNOWLEDGEMENTS}

This work was supported by a seed grant from the University of Chicago Brain Research Foundation,
R03-DA024197, R01-AA013746, Cancer Center P30-CA14599, National Center for Research Resources Grant UL1RR024999. We thank Dan Fitzgerald and Adrienne Dellinger for their assistance in stimulus preparation.

\section{DISCLOSURES}

The authors declare that, except for income received from their primary employer, no financial support or compensation has been received from any individual or corporate entity over the past 3 years for research or professional service and there are no personal financial holdings that could be perceived as constituting a potential conflict of interest.

\section{REFERENCES}

Baer JS, Lichtenstein E (1988). Classification and prediction of smoking relapse episodes: an exploration of individual differences. J Consult Clin Psychol 56: 104-110.

Barson JR, Carr AJ, Soun JE, Sobhani NC, Leibowitz SF, Hoebel BG (2009). Opioids in the nucleus accumbens stimulate ethanol intake. Physiol Behav 98: 453-459.

Boileau I, Assaad JM, Pihl RO, Benkelfat C, Leyton M, Diksic M et al (2003). Alcohol promotes dopamine release in the human nucleus accumbens. Synapse 49: 226-231.

Bragulat V, Dzemidzic M, Talavage T, Davidson D, O'Connor SJ, Kareken DA (2008). Alcohol sensitizes cerebral responses to the odors of alcoholic drinks: an fMRI study. Alcohol Clin Exp Res 32: 1124-1134.

Brody AL (2006). Functional brain imaging of tobacco use and dependence. J Psychiatr Res 40: 404-418.

Brody AL, Mandelkern MA, London ED, Childress AR, Lee GS, Bota RG et al (2002). Brain metabolic changes during cigarette craving. Arch Gen Psychiatry 59: 1162-1172.

Brody AL, Mandelkern MA, Olmstead RE, Jou J, Tiongson E, Allen $\mathrm{V}$ et al (2007). Neural substrates of resisting craving during cigarette cue exposure. Biol Psychiatry 62: 642-651.

Bustamante D, Quintanilla ME, Tampier L, Gonzalez-Lira V, Israel Y, Herrera-Marschitz M (2008). Ethanol induces stronger dopamine release in nucleus accumbens (shell) of alcoholpreferring (bibulous) than in alcohol-avoiding (abstainer) rats. Eur J Pharmacol 591: 153-158.

Cahalan D, Cisin IH (1968). American drinking practices: summary of findings from a national probability sample. II. Measurement of massed versus spaced drinking. Q J Stud Alcohol 29: 642-656.

Cox LS, Tiffany ST, Christen AG (2001). Evaluation of the brief questionnaire of smoking urges (QSU-brief) in laboratory and clinical settings. Nicotine Tob Res 3: 7-16.

Dani JA, Harris RA (2005). Nicotine addiction and comorbidity with alcohol abuse and mental illness. Nat Neurosci 8: 1465-1470.

David SP, Munafo MR, Johansen-Berg H, Smith SM, Rogers RD, Matthews PM et al (2005). Ventral striatum/nucleus accumbens activation to smoking-related pictorial cues in smokers and nonsmokers: a functional magnetic resonance imaging study. Biol Psychiatry 58: 488-494.

Dawson DA (2000). Drinking as a risk factor for sustained smoking. Drug Alcohol Depend 59: 235-249.

DiFranza JR, Guerrera MP (1990). Alcoholism and smoking. J Stud Alcohol 51: 130-135.

Due DL, Huettel SA, Hall WG, Rubin DC (2002). Activation in mesolimbic and visuospatial neural circuits elicited by smoking cues: evidence from functional magnetic resonance imaging. Am J Psychiatry 159: 954-960. 
Epstein AM, Sher TG, Young MA, King AC (2007). Tobacco chippers show robust increases in smoking urge after alcohol consumption. Psychopharmacology (Berl) 190: 321-329.

Ernst M, Bolla K, Mouratidis M, Contoreggi C, Matochik JA, Kurian V et al (2002). Decision-making in a risk-taking task: a PET study. Neuropsychopharmacology 26: 682-691.

Everitt BJ, Robbins TW (2005). Neural systems of reinforcement for drug addiction: from actions to habits to compulsion. Nat Neurosci 8: 1481-1489.

First MB, Spitzer RL, Gibbon M, Williams JBW (1995). Structured Clinical Interview for DSM-IV Axis I Disorders. Biometrics Research Department-Patient Edition (SCID-I/P, Version 2.0): New York, NY.

Franklin TR, Wang Z, Wang J, Sciortino N, Harper D, Li Y et al (2007). Limbic activation to cigarette smoking cues independent of nicotine withdrawal: a perfusion fMRI study. Neuropsychopharmacology 32: 2301-2309.

Frezza M, di Padova C, Pozzato G, Terpin M, Baraona E, Lieber CS (1990). High blood alcohol levels in women. The role of decreased gastric alcohol dehydrogenase activity and first-pass metabolism. N Engl J Med 322: 95-99.

Genovese CR, Lazar NA, Nichols T (2002). Thresholding of statistical maps in functional neuroimaging using the false discovery rate. Neuroimage 15: 870-878.

Gilbert DG, Rabinovich NE (2003). The Emotional Image Series, Version 1.1 Manual. In. Department of Psychology, Southern Illinois University: Carbondale, IL.

Gilman JM, Ramchandani VA, Davis MB, Bjork JM, Hommer DW (2008). Why we like to drink: a functional magnetic resonance imaging study of the rewarding and anxiolytic effects of alcohol. J Neurosci 28: 4583-4591.

Harrison EL, Desai RA, McKee SA (2008). Nondaily smoking and alcohol use, hazardous drinking, and alcohol diagnoses among young adults: findings from the NESARC. Alcohol Clin Exp Res 32: 2081-2087.

Heatherton TF, Kozlowshi LT, Frecker RC, Fagerström K (1991). The Fagerström Test for Nicotine Dependence: a revision of the Fagerström tolerance questionnaire. Br J Addict 86: 1119-1127.

Honkanen A, Ahtee L, Korpi ER (1997). Voluntary alcohol drinking selectively accelerates dopamine release in the ventral striatum as reflected by 3-methoxytyramine levels. Brain Res 774: $207-210$.

Kalivas PW, Volkow ND (2005). The neural basis of addiction: a pathology of motivation and choice. Am J Psychiatry 162: 14031413.

Kareken DA, Claus ED, Sabri M, Dzemidzic M, Kosobud AE, Radnovich AJ et al (2004). Alcohol-related olfactory cues activate the nucleus accumbens and ventral tegmental area in high-risk drinkers: preliminary findings. Alcohol Clin Exp Res 28: $550-557$.

King AC, Epstein AM (2005). Alcohol dose-dependent increases in smoking urge in light smokers. Alcohol Clin Exp Res 29: 547-552.

King AC, Epstein AM, Conrad MF, McNamara PJ, Cao D (2008). Sex differences in the relationship between alcohol-associated smoking urge and behavior: a pilot study. Am J Addict 17: 347-353.

King AC, McNamara PJ, Conrad MF, Cao D (2009). Alcoholinduced increases in smoking behavior for nicotinized and denicotinized cigarettes in men and women. Psychopharmacology 207: 107-117.

Knutson B, Gibbs SE (2007). Linking nucleus accumbens dopamine and blood oxygenation. Psychopharmacology (Berl) 191: 813-822.

Koob GF, Le Moal M (1997). Drug abuse: hedonic homeostatic dysregulation. Science 278: 52-58.

Koob GF, Le Moal M (2001). Drug addiction, dysregulation of reward, and allostasis. Neuropsychopharmacology 24: 97-129.
London ED, Ernst M, Grant S, Bonson K, Weinstein A (2000). Orbitofrontal cortex and human drug abuse: functional imaging. Cereb Cortex 10: 334-342.

Martinez D, Slifstein M, Broft A, Mawlawi O, Hwang DR, Huang Y et al (2003). Imaging human mesolimbic dopamine transmission with positron emission tomography. Part II: amphetamine-induced dopamine release in the functional subdivisions of the striatum. J Cereb Blood Flow Metab 23: 285-300.

McBride D, Barrett SP, Kelly JT, Aw A, Dagher A (2006). Effects of expectancy and abstinence on the neural response to smoking cues in cigarette smokers: an fMRI study. Neuropsychopharmacology 31: 2728-2738.

McClernon FJ, Gilbert DG (2004). Human functional neuroimaging in nicotine and tobacco research: basics, background, and beyond. Nicotine Tob Res 6: 941-959.

McClernon FJ, Hiott FB, Huettel SA, Rose JE (2005). Abstinenceinduced changes in self-report craving correlate with event-related FMRI responses to smoking cues. Neuropsychopharmacology 30: 1940-1947.

McClernon FJ, Kozink RV, Lutz AM, Rose JE (2008a). 24-h smoking abstinence potentiates fMRI-BOLD activation to smoking cues in cerebral cortex and dorsal striatum. Psychopharmacology (Berl) 204: 25-35.

McClernon FJ, Kozink RV, Rose JE (2008b). Individual differences in nicotine dependence, withdrawal symptoms, and sex predict transient fMRI-BOLD responses to smoking cues. Neuropsychopharmacology 33: 2148-2157.

McKee SA, Hinson R, Rounsaville D, Petrelli P (2004). Survey of subjective effects of smoking while drinking among college students. Nicotine Tob Res 6: 111-117.

Mitchell SH, de Wit H, Zacny JP (1995). Effects of varying ethanol dose on cigarette consumption in healthy normal volunteers. Behav Pharmacol 6: 359-365.

Niaura RS, Rohsenow DJ, Binkoff JA, Monti PM, Pedraza M, Abrams DB (1988). Relevance of cue reactivity to understanding alcohol and smoking relapse. J Abnorm Psychol 97: 133-152.

O’Doherty J, Kringelbach ML, Rolls ET, Hornak J, Andrews C (2001). Abstract reward and punishment representations in the human orbitofrontal cortex. Nat Neurosci 4: 95-102.

Phan KL, Angstadt M, Golden J, Onyewuenui I, Povpovska A, de Wit H (2008). Cannabinoid modulation of amygdala reactivity to social signals of threat in humans. J Neurosci 28: 2313-2319.

Robinson TE, Berridge KC (1993). The neural basis of drug craving: an incentive-sensitization theory of addiction. Brain Res Brain Res Rev 18: 247-291.

Rogers RD, Owen AM, Middleton HC, Williams EJ, Pickard JD, Sahakian BJ et al (1999). Choosing between small, likely rewards and large, unlikely rewards activates inferior and orbital prefrontal cortex. J Neurosci 19: 9029-9038.

SAMHSA (2005). National Survey on Drug Use and Health. Office of Applied Studies: Bethesda, MD.

Sayette MA, Martin CS, Wertz JM, Perrott MA, Peters AR (2005). The effects of alcohol on cigarette craving in heavy smokers and tobacco chippers. Psychol Addict Behav 19: 263-270.

Sayette MA, Shiffman S, Tiffany ST, Niaura RS, Martin CS, Shadel WG (2000). The measurement of drug craving. Addiction 95(Suppl 2): S189-S210.

Schott BH, Minuzzi L, Krebs RM, Elmenhorst D, Lang M, Winz OH et al (2008). Mesolimbic functional magnetic resonance imaging activations during reward anticipation correlate with reward-related ventral striatal dopamine release. J Neurosci 28: 14311-14319.

Shiffman S (1989). Tobacco 'chippers' - individual differences in tobacco dependence. Psychopharmacology (Berl) 97: 539-547.

Shiffman S (2009). Light and intermittent smokers: background and perspective. Nicotine Tob Res 11: 122-125. 
Shiffman S, Fischer LB, Zettler-Segal M, Benowitz NL (1990). Nicotine exposure among nondependent smokers. Arch Gen Psychiatry 47: 333-336.

Shiffman S, Paty JA, Gnys M, Kassel JD, Elash C (1995). Nicotine withdrawal in chippers and regular smokers: subjective and cognitive effects. Health Psychol 14: 301-309.

Smolka MN, Buhler M, Klein S, Zimmermann U, Mann K, Heinz A et al (2006). Severity of nicotine dependence modulates cueinduced brain activity in regions involved in motor preparation and imagery. Psychopharmacology (Berl) 184: 577-588.

Sobell LC, Maisto SA, Sobell MB, Cooper AM (1979). Reliability of alcohol abusers' self-reports of drinking behavior. Behav Res Ther 17: 157-160.

Sobell LC, Sobell MB (1995). Alcohol Timeline Follow-Back Users' Manual. Addiction Research Foundation: Toronto, ON.

Sobell LC, Sobell MB, Kozlowski LT, Toneatto T (1990). Alcohol or tobacco research versus alcohol and tobacco research. $\mathrm{Br} J$ Addict 85: 263-269.

Sutker PB, Tabakoff B, Goist Jr KC, Randall CL (1983). Acute alcohol intoxication, mood states and alcohol metabolism in women and men. Pharmacol Biochem Behav 18(Suppl 1): 349-354.

Tzourio-Mazoyer N, Landeau B, Papathanassiou D, Crivello F, Etard O, Delcroix N et al (2002). Automated anatomical labeling of activations in SPM using a macroscopic anatomical parcellation of the MNI MRI single-subject brain. Neuroimage 15: 273-289.

Walter B, Blecker C, Kirsch P, Sammer G, Schienle A, Stark R et al (2003). MARINA: an easy to use tool for the creation of MAsks for Interest Analyses. Neuroimage 19: S47.

Wang Z, Faith M, Patterson F, Tang K, Kerrin K, Wileyto EP et al (2007). Neural substrates of abstinence-induced cigarette cravings in chronic smokers. J Neurosci 27: 14035-14040.

Wilson SJ, Sayette MA, Delgado MR, Fiez JA (2005). Instructed smoking expectancy modulates cue-elicited neural activity: a preliminary study. Nicotine Tob Res 7: 637-645.

World Health Organization (2007). http://www.who.int/.

Wrase J, Grusser SM, Klein S, Diener C, Hermann D, Flor $\mathrm{H}$ et al (2002). Development of alcohol-associated cues and cue-induced brain activation in alcoholics. Eur Psychiatry 17: 287-291. 